\title{
Erythrocyte trans-fatty acids, type 2 diabetes and cardiovascular risk factors in middle-aged and older Chinese individuals
}

\author{
D. X. Yu • Q. Sun • X. W. Ye • A. Pan • G. Zong • \\ Y. H. Zhou • H. X. Li • F. B. Hu • X. Lin
}

Received: 23 April 2012 / Accepted: 10 July 2012 /Published online: 12 August 2012

(C) Springer-Verlag 2012

\begin{abstract}
Aims/hypothesis Few data are available about intakes and food sources of trans-fatty acids (TFAs) or their associations with cardiometabolic outcomes in Asian people who consume a prudent diet but are experiencing rapid nutritional transitions. We aimed to investigate the relationships between TFA biomarkers and type 2 diabetes and cardiovascular risk factors in Chinese individuals.

Methods Erythrocyte fatty acids were measured by gas chromatography among 3,107 men and women (5070 years) recruited from urban and rural areas in Beijing and Shanghai, China.
\end{abstract}

Electronic supplementary material The online version of this article (doi:10.1007/s00125-012-2674-2) contains peer-reviewed but unedited supplementary material, which is available to authorised users.

D. X. Yu X. W. Ye $\cdot$ G. Zong • Y. H. Zhou $\cdot$ H. X. Li $\cdot$ X. Lin $(\bowtie)$

Key Laboratory of Nutrition and Metabolism, Institute for Nutritional Sciences, Shanghai Institutes for Biological Sciences, Chinese Academy of Sciences and Graduate School of Chinese Academy of Sciences,

294 Taiyuan Rd,

Shanghai 200031, People's Republic of China

e-mail: xlin@sibs.ac.cn

Q. Sun · A. Pan · F. B. Hu

Department of Nutrition, Harvard School of Public Health,

Boston, MA, USA

Q. Sun · F. B. Hu

Channing Division of Network Medicine,

Department of Medicine,

Brigham and Women's Hospital and Harvard Medical School,

Boston, MA, USA

F. B. $\mathrm{Hu}$

Department of Epidemiology, Harvard School of Public Health, Boston, MA, USA
Results Total trans-18:1 and two trans-18:2 isomers were detected and accounted for $0.37 \%$ of the total fatty acids in the erythrocytes. Concentrations of TFAs were higher in women than men, and in urban than rural residents. Of the TFAs, trans-18:1, but not trans-18:2, showed a modest association with dairy consumption $(\beta=0.27)$, but not with other foods. After adjustment for BMI, social-demographic, lifestyle and dietary factors and other TFAs, erythrocyte trans-18:1 was shown to be associated with a lower risk of type 2 diabetes (OR comparing extreme [first and fourth] quartiles $0.68,95 \%$ CI $0.48,0.97, p_{\text {trend }}=0.02$ ), as well as $20-50 \%$ lower odds of central obesity, dyslipidaemia, hyperglycaemia, insulin resistance and chronic inflammation. In contrast, trans-18:2 fatty acids were positively associated with high triacylglycerol $\left(p_{\text {trend }}<0.001\right)$ and LDLcholesterol $\left(p_{\text {trend }}=0.03\right)$ levels, but not with diabetes and other cardiometabolic risk factors.

Conclusions/interpretation Among middle-aged and older Chinese individuals with overall low erythrocyte TFAs levels, trans-18:1 might serve as a marker of dairy intake. Higher trans-18:1 levels were associated with a lower risk of type 2 diabetes, whereas higher trans-18:2 levels were associated with dyslipidaemia.

Keywords Biomarkers $\cdot$ Diet $\cdot$ Dyslipidaemia $\cdot$ trans-Fatty acids · Type 2 diabetes

$\begin{array}{ll}\text { Abbreviations } \\ \text { CHS } & \text { Cardiovascular Health Study } \\ \text { FAME } & \text { Fatty acid methyl ester } \\ \text { FFQ } & \text { Food-frequency questionnaire } \\ \text { GI } & \text { Glycaemic index } \\ \text { HOMA-IR } & \text { HOMA of insulin resistance } \\ \text { hs-CRP } & \text { High-sensitivity C-reactive protein } \\ \text { iTFA } & \text { Industrially produced trans-fatty acid }\end{array}$




$\begin{array}{ll}\text { NHS } & \text { Nurses' Health Study } \\ \text { PHVO } & \text { Partially hydrogenated vegetable oil } \\ \text { rTFA } & \text { Ruminant trans-fatty acid } \\ \text { TFA } & \text { trans-Fatty acid } \\ \text { VA } & \text { Vaccenic acid }\end{array}$

\section{Introduction}

In recent decades, many Asian countries including China have been undergoing rapid nutritional and lifestyle transitions. Excessive energy intakes and an increased consumption of animal foods are likely to be the major drivers of the current chronic disease epidemics seen in developing countries [1]. According to the 1992-2002 China Health and Nutrition Surveys, meat and dairy intakes were almost doubled and energy intake from fat and animal foods increased from $22.0 \%$ to $29.8 \%$ and from $9.3 \%$ to $13.7 \%$, respectively, over this period [2]. Consequently, both the quantity and the quality of dietary fats have been concomitantly altered with the shift from a traditional to a Westernised dietary pattern. Meanwhile, metabolic disorders, such as central obesity, dyslipidaemia, insulin resistance and type 2 diabetes, have now become major public health challenges in China. It is thus critical to elucidate the role of dietary factors, including dietary fatty acids, in the development of cardiometabolic outcomes in Chinese individuals.

trans-Fatty acids (TFAs) have been demonstrated to have adverse effects on cardiometabolic diseases [3, 4]. A metaanalysis of 13 clinical trials has shown that iso-energy substitution of TFAs for other fatty acids increased triacylglycerol levels and total to HDL-cholesterol ratios [5]. TFAs, however, have two primary food origins: industrially produced partially hydrogenated vegetable oils (PHVOs), or industrially produced trans-fatty acids (iTFAs), and ruminant fats (naturally produced ruminant TFAs [rTFAs]). Most previous studies have focused on total TFAs or iTFAs, the dominant TFAs in the Western diet [3], whereas results with respect to rTFAs among populations with a low overall iTFA consumption are scarce and inconsistent $[6,7]$.

Food-frequency questionnaires (FFQs) have been commonly used to assess TFA consumption $[8,9]$. This method is, however, subject to measurement errors caused by recall bias and inaccurate estimation of food contents for TFAs, which vary by brand, degree of hydrogenation, season and even cow feed $[10,11]$. Therefore, especially for regions or countries without comprehensive and accurate food composition data, such as China, objective markers of TFA intake are preferred because diet is the only source of these fatty acids in human tissues [3].

So far, only a few studies have used measured TFA biomarkers to examine the specific effect of individual trans-isomers. For instance, in the Cardiovascular Health Study (CHS), trans-18:1 isomers were inversely and trans18:2 isomers were positively associated with an increased risk of $\mathrm{CHD}$, although no significant association was detected for total TFA in plasma phospholipids [12]. Moreover, trans-16:1n-7, a marker of dairy fat, was associated with a lower incidence of type 2 diabetes and favourable metabolic traits [13]. To date, however, few data have been available regarding TFA status and related health consequences in Chinese individuals, whose TFA consumption is typically low. We therefore measured erythrocyte fatty acids using gas chromatography and determined various TFA isomers in relation to type 2 diabetes and multiple cardiovascular risk factors among 50-70-year-old Chinese men and women.

\section{Methods}

Study population A population-based sample was obtained from the Nutrition and Health of Aging Population in China study, which recruited 1,458 men and 1,831 women aged 50-70 years to examine environmental and genetic factors in relation to chronic diseases [14]. Beijing and Shanghai were selected as representative cities in northern and southern China, respectively. For each city, one rural county and two urban districts were sampled to represent low, middle or high socioeconomic level. The detailed study design and inclusion/exclusion criteria have been described elsewhere [14]. After excluding 31 individuals with insufficient erythrocyte samples and 151 individuals with an extreme total energy intake $(<3,347$ or $>16,736 \mathrm{~kJ} /$ day for men and $<2,092$ or $>14,644 \mathrm{~kJ} /$ day for women), a total of 3,107 eligible participants were included in the current analyses. The study was approved by the institutional review board of the Institute for Nutritional Sciences, and written informed consent was provided by all participants.

Data and sample collection A standardised questionnaire was used during home interviews to collect information on social demographics, health status, smoking (current, former or never), alcohol consumption (yes/no) and physical activity [14]. A family history of chronic diseases was considered positive if a parent or first-degree sibling had CHD, stroke, type 2 diabetes or hypertension. Educational level was classified according to self-reported school years (0-6, 7-9, $\geq 10$ years). Physical activity was categorised as low, moderate and high from the sum of metabolic equivalent minutes expended per week [15].

Food intakes were obtained by a 74-item FFQ modified from the 2002 China Health and Nutrition Surveys [16]. The FFQ used in the national survey has been validated and widely used among studies in China $[17,18]$. There were five 
questions regarding dairy food consumption (milk, milk powder, yogurt, ice cream and other dairy products). Intakes of energy, macronutrients and fibre were calculated by converting frequencies and serving sizes of specific foods into grams per day and linking these to the Chinese Food Composition Table, except for TFAs, which were not included in this database [19]. The glycaemic indexes (GIs) of all carbohydrate-containing foods were extracted from the International Tables of Glycemic Index and Glycemic Load Values [20], and the weighted average was calculated to obtain dietary GI.

After an overnight fast, trained physicians and staff members gave all participants a physical examination. Measurements of height, weight, waist circumference and blood pressure have been described elsewhere [14]. Blood samples were collected using EDTA as an anticoagulant, centrifuged to separate the plasma, buffy coat and erythrocytes and then stored at $-80^{\circ} \mathrm{C}$. Measurement of plasma fasting glucose, insulin, triacylglycerol, lipoproteins and high-sensitivity Creactive protein (hs-CRP) has also previously been described $[14,15]$.

Outcome assessment Type 2 diabetes was defined as a fasting plasma glucose $\geq 7.0 \mathrm{mmol} / \mathrm{l}$, prior diagnosed diabetes mellitus or the self-reported use of antidiabetic medications [21]. Other metabolic disorders were defined according to the updated National Cholesterol Education Program Adult Treatment Panel III criteria for Asian-Americans [22]. Specifically, central obesity was defined as a waist circumference $\geq 90 \mathrm{~cm}$ in men and $\geq 80 \mathrm{~cm}$ in women; hyperglycaemia was defined as a fasting plasma glucose $\geq 5.6 \mathrm{mmol} / 1$; high blood pressure was defined as blood pressure $\geq 130$ / $85 \mathrm{mmHg}$ or current use of antihypertensive medication; hypertriacylglycerolaemia was defined as a triacylglycerol value $\geq 1.7 \mathrm{mmol} / 1$; and low HDL-cholesterol was defined as an HDL-cholesterol level $<1.03 \mathrm{mmol} / 1$ in men and $<1.30 \mathrm{mmol} / 1$ in women. The HOMA model of insulin resistance (HOMA-IR) was computed using updated methods (www.dtu.ox.ac.uk). Insulin resistance was defined as a HOMA-IR higher than $75 \%$ level in non-diabetic participants (2.08 in this study). Chronic inflammation was defined as an hs-CRP value $>3 \mathrm{mg} / \mathrm{l}$, according to the American Heart Association criterion [23].

Erythrocyte fatty acid measurement Fatty acids were measured following the protocol used in the Nurses' Health Study (NHS), with minor modification [24]. Briefly, erythrocytes $(400 \mu \mathrm{l})$ were mixed with isopropanol, hexane and internal standard (1 $\mu \mathrm{g}$ 1,2-dihenarachidoyl-sn-glycero-3phosphocholine dissolved in $1 \mathrm{ml}$ chloroform), followed by methylation (methanol and sulphuric acid). After $2.5 \mathrm{~h}$ of incubation, fatty acid methyl esters (FAMEs) were twice extracted by hexane, evaporated under nitrogen and suspended in isooctane.
FAMEs were analysed by gas chromatography (Agilent $6890 \mathrm{GC}$ with flame ionisation detector; SP-2560 capillary column: $100 \mathrm{~m} \times 0.25 \mathrm{~mm}$ i.d. $\times 0.2 \mu \mathrm{m}$ film; Supelco, Bellefonte, PA, USA; carrier gas nitrogen). Esters were identified by electron impact mass spectrometry, and methane was used as a reagent gas for positive chemical ionisation (Agilent 6890N-5975B; Supelco). Quantification was calibrated by the internal standard and an equal-weight FAMEs mixture (GLC-569B; Nu-Chek Prep, Elysian, MN, USA). Chromatography conditions have been previously reported [25]. The relative amount of each fatty acid (\% of total fatty acids) was quantified. Within-run coefficients of variations were $14.5 \%$ for trans-18:1 and $21.4 \%$ for trans18:2 fatty acids.

Statistical analysis Multiple linear regressions were used to evaluate associations between erythrocyte TFAs and major sources of dietary trans-fats in traditional Chinese diets (e.g. dairy products, ruminant meats, fried foods and cooking oil). Multivariable logistic regressions were applied to estimate the risks of metabolic disorders, adjusting for potential confounders, including age (continuous), sex, northern or southern geographical region, urban or rural residence, family history of chronic diseases, educational attainment, physical activity level, smoking, alcohol consumption, daily energy intake (continuous), consumption of carbohydrate and protein (\% of energy) and BMI (continuous). Dietary factors including GI, red meat, vegetables, fruit and dietary fibre were further controlled for in the models (continuous and energy-adjusted by residual method) [26]. Values of $p$ for trend were determined by assuming median values of quartiles as continuous variables. Stratified analyses were conducted according to sex, age, region, physical activity, waist circumference and BMI. Interactions were calculated using the likelihood ratio test. All statistical analyses were performed using Stata 9.2 (College Station, TX, USA) with a two-tailed $p<0.05$.

\section{Results}

Erythrocyte TFA concentrations and related food sources A total of three TFA isomers were quantified in erythrocytes. The mean content as a percentage of total fatty acids was 0.20 for total trans-18:1 (isomers with a different doublebond location could not be further separated because of low levels), and the median levels were 0.093 for trans $-18: 2 n-6$ $9 \mathrm{c} 12 \mathrm{t}$ and 0.062 for trans-18:2n-6 9t12c (Table 1). Female and northern residents had slightly but significantly higher total TFAs and trans-18:1 than their male and southern counterparts, whereas urban participants had significantly higher levels of all three detected TFAs than rural participants. 
Table 1 Erythrocyte TFA content and distribution ${ }^{\mathrm{a}}$

Data (\% of total fatty acids) are means \pm SD or geometric mean $(95 \% \mathrm{CI})$

${ }^{\mathrm{b}} p$ significant for comparison by sex or region

\begin{tabular}{lllll}
\hline Variable & Total TFAs & trans-18:1 & $18: 2 \mathrm{n}-69 \mathrm{c} 12 \mathrm{t}$ & $18: 2 \mathrm{n}-69 \mathrm{t} 12 \mathrm{c}$ \\
\hline Total participants & $0.37 \pm 0.11$ & $0.20 \pm 0.06$ & $0.093(0.092,0.094)$ & $0.062(0.061,0.063)$ \\
Female $(n=1,778)$ & $0.38 \pm 0.12^{\mathrm{b}}$ & $0.21 \pm 0.06^{\mathrm{b}}$ & $0.095(0.093,0.096)$ & $0.063(0.062,0.064)$ \\
Male $(n=1,329)$ & $0.36 \pm 0.11^{\mathrm{b}}$ & $0.20 \pm 0.06^{\mathrm{b}}$ & $0.091(0.090,0.093)$ & $0.060(0.059,0.062)$ \\
Northern China $(n=1,515)$ & $0.38 \pm 0.10^{\mathrm{b}}$ & $0.21 \pm 0.06^{\mathrm{b}}$ & $0.095(0.094,0.097)$ & $0.064(0.063,0.065)$ \\
Southern China $(n=1,592)$ & $0.36 \pm 0.12^{\mathrm{b}}$ & $0.20 \pm 0.07^{\mathrm{b}}$ & $0.091(0.090,0.093)$ & $0.060(0.059,0.061)$ \\
Urban $(n=1,574)$ & $0.41 \pm 0.12^{\mathrm{b}}$ & $0.22 \pm 0.06^{\mathrm{b}}$ & $0.103(0.101,0.104)^{\mathrm{b}}$ & $0.071(0.069,0.072)^{\mathrm{b}}$ \\
Rural $(n=1,533)$ & $0.33 \pm 0.09^{\mathrm{b}}$ & $0.18 \pm 0.06^{\mathrm{b}}$ & $0.085(0.083,0.086)^{\mathrm{b}}$ & $0.054(0.053,0.055)^{\mathrm{b}}$ \\
\hline
\end{tabular}

To determine potential food sources for erythrocyte TFAs, multiple linear regressions were applied with adjustment for age, sex, north/south region, urban/rural location, education, smoking and drinking habits, physical activity, total energy intake and BMI. Levels of trans-18:1 were significantly associated with dairy food consumption: each SD of dairy intake was associated with a 0.27 SD higher concentration of trans-18:1 $(p<0.001)$, whereas the associations with other foods, including ruminant meats, fried foods and cooking oil, were not significant. In addition, no associations were detected between trans-18:2 isomers and dairy consumption.

ORs of metabolic disorders according to trans-18:1 levels Participants with higher quartiles of erythrocyte trans18:1 were more likely to be female, northern or urban residents, have a positive family history of chronic diseases and have higher levels of educational attainment and physical activity, but were less likely to be current smokers (see electronic supplementary material [ESM] Table 1). Participants mostly ate a traditional Chinese diet, characterised as high carbohydrate and low fat intakes (mean \pm SD $[\%$ of energy]: $59.3 \pm 9.5 \%$ and $28.6 \pm 8.2 \%$, respectively) as well as high vegetable and fruit, but low red meat, consumption $(547 \pm 308 \mathrm{~g} /$ day and $45.9 \pm 45.0 \mathrm{~g} /$ day, respectively). However, individuals with higher trans-18:1 levels tended to consume more protein, fat, red meat, dietary fibre, fruits and all kinds of dairy products. Meanwhile, they were more likely to have a lower energy and carbohydrate intake and a lower dietary GI.

From the lowest to highest quartile of trans-18:1, the ORs and 95\% CIs were 1.00 (reference), $0.75(0.55,1.02)$, $0.59(0.43,0.81)$ and $0.61(0.44,0.85)$ for type 2 diabetes (Model 2; $p_{\text {trend }}=0.002$ ) (Table 2). This inverse association was independent of social-demographic and lifestyle variables, daily energy and macronutrients intakes and BMI. Further adjustment for dietary GI, red meat, vegetable, fruit and fibre intake and erythrocyte trans-18:2 levels did not change the results (Model 3, OR [95\% CI] 0.68 [0.48, 0.97] comparing extreme (first and fourth) quartiles; $p_{\text {trend }}=0.02$ ).

Similarly, participants in the highest trans-18:1 quartile had significantly lower odds of central obesity (OR [95\%
CI] $0.66[0.46,0.94])$, elevated triacylglycerol $(0.50[0.38$, $0.65])$ and fasting glucose $(0.80[0.62,1.02])$ levels, and reduced HDL-cholesterol $(0.56$ [0.44, 0.72]), insulin resistance $(0.62[0.47,0.81])$ and chronic inflammation $(0.69$ $[0.49,0.97])$ compared with those in the lowest quartile (Model 3; all $p_{\text {trend }}<0.05$ ). However, the significant associations between erythrocyte trans-18:1 levels and elevated blood pressure were attenuated after adjusting for BMI and lifestyle and/or dietary covariates $\left(p_{\text {trend }}<0.001\right.$ in Model 1; $p_{\text {trend }}=0.23$ in Model 3, respectively).

ORs of metabolic disorders according to trans-18:2 levels In contrast to trans-18:1, increasing quartiles of trans-18:2 (the sum of trans-18:2n-6 9c12t and trans-18:2n-6 9t12c) were not significantly associated with risk of type 2 diabetes, central obesity, hyperglycaemia, high blood pressure, insulin resistance, chronic inflammation or low HDLcholesterol levels, but were positively associated with hypertriacylglycerolaemia and elevated total cholesterol and LDL-cholesterol levels (Table 3, Model 2). Comparing the highest with the lowest quartiles, ORs $(95 \% \mathrm{CI})$ were $1.91(1.43,2.54), 1.32(1.02,1.72)$ and $1.33(1.05,1.68)$, respectively. Further adjustment for erythrocyte saturated fatty acids did not substantially alter these associations (Model 3).

Secondary analyses In sensitivity analyses, after we had excluded participants with previously diagnosed diabetes $(n=245)$ or any chronic diseases such as hypertension, high cholesterol levels and diabetes $(n=1,634)$, trans-18:1 levels remained inversely associated with newly diagnosed diabetes; the OR (95\% CI) across extreme quartiles was 0.35 $(0.15,0.79)\left(p_{\text {trend }}=0.01\right)$. Meanwhile, further adjustment for dairy intake or plasma 25-hydroxyvitamin D levels did not abolish the inverse associations between trans-18:1 and diabetes (ORs $[95 \% \mathrm{CI}]$ across extreme quartiles $0.60[0.42$, $0.86], p_{\text {trend }}=0.003$ and $0.69[0.48,0.98], p_{\text {trend }}=0.03$, respectively). However, additionally controlling for waist circumference slightly attenuated the inverse association: ORs (95\% CI) across trans-18:1 quartiles were 1.00 (reference), $0.74(0.54,1.02), 0.59(0.43,0.83)$ and $0.72(0.51,1.03)$ $\left(p_{\text {trend }}=0.05\right)$, respectively. 
Table 2 ORs for type 2 diabetes and metabolic disorders according to quartiles of trans-18:1 fatty acid

\begin{tabular}{|c|c|c|c|c|c|}
\hline trans $-18: 1$ & Q1 (median 0.138) & Q2 (median 0.177) & Q3 (median 0.214) & Q4 (median 0.270) & $p$ for trend \\
\hline Type 2 diabetes ${ }^{\mathrm{a}}$ & 116 & 111 & 97 & 101 & \\
\hline Model 1 & 1 & $0.81(0.61,1.08)$ & $0.65(0.48,0.88)$ & $0.65(0.48,0.88)$ & 0.003 \\
\hline Model 2 & 1 & $0.75(0.55,1.02)$ & $0.59(0.43,0.81)$ & $0.61(0.44,0.85)$ & 0.002 \\
\hline Model 3 & 1 & $0.75(0.55,1.03)$ & $0.58(0.42,0.81)$ & $0.68(0.48,0.97)$ & 0.02 \\
\hline Central obesity $^{\mathrm{a}}$ & 381 & 429 & 368 & 322 & \\
\hline Model 1 & 1 & $1.11(0.90,1.38)$ & $0.72(0.58,0.90)$ & $0.47(0.38,0.59)$ & $<0.0001$ \\
\hline Model 2 & 1 & $1.15(0.83,1.60)$ & $0.87(0.62,1.21)$ & $0.67(0.47,0.95)$ & 0.004 \\
\hline Model 3 & 1 & $1.14(0.82,1.60)$ & $0.85(0.60,1.19)$ & $0.66(0.46,0.94)$ & 0.004 \\
\hline Hypertriacylglycerolaemia $^{a}$ & 205 & 215 & 182 & 154 & \\
\hline Model 1 & 1 & $0.92(0.73,1.16)$ & $0.68(0.54,0.87)$ & $0.48(0.38,0.62)$ & $<0.0001$ \\
\hline Model 2 & 1 & $0.93(0.73,1.18)$ & $0.75(0.59,0.96)$ & $0.58(0.44,0.75)$ & $<0.0001$ \\
\hline Model 3 & 1 & $0.91(0.72,1.16)$ & $0.70(0.55,0.91)$ & $0.50(0.38,0.65)$ & $<0.0001$ \\
\hline Low HDL-cholesterol ${ }^{\mathrm{a}}$ & 372 & 333 & 321 & 290 & \\
\hline Model 1 & 1 & $0.75(0.61,0.92)$ & $0.67(0.54,0.83)$ & $0.47(0.38,0.59)$ & $<0.0001$ \\
\hline Model 2 & 1 & $0.73(0.59,0.91)$ & $0.74(0.59,0.92)$ & $0.57(0.45,0.72)$ & $<0.0001$ \\
\hline Model 3 & 1 & $0.73(0.58,0.91)$ & $0.73(0.58,0.92)$ & $0.56(0.44,0.72)$ & $<0.0001$ \\
\hline Hyperglycaemia $^{a}$ & 307 & 334 & 320 & 295 & \\
\hline Model 1 & 1 & $1.00(0.81,1.24)$ & $0.86(0.69,1.07)$ & $0.75(0.60,0.94)$ & 0.004 \\
\hline Model 2 & 1 & $0.96(0.77,1.20)$ & $0.87(0.70,1.09)$ & $0.80(0.63,1.00)$ & 0.04 \\
\hline Model 3 & 1 & $0.97(0.78,1.21)$ & $0.86(0.68,1.08)$ & $0.80(0.62,1.02)$ & 0.04 \\
\hline High blood pressure ${ }^{a}$ & 575 & 575 & 530 & 519 & \\
\hline Model 1 & 1 & $0.97(0.77,1.23)$ & $0.72(0.57,0.91)$ & $0.71(0.56,0.90)$ & 0.0007 \\
\hline Model 2 & 1 & $1.00(0.78,1.28)$ & $0.80(0.63,1.03)$ & $0.88(0.68,1.14)$ & 0.20 \\
\hline Model 3 & 1 & $0.99(0.77,1.27)$ & $0.79(0.62,1.01)$ & $0.89(0.68,1.16)$ & 0.23 \\
\hline Insulin resistance ${ }^{\mathrm{a}}$ & 229 & 244 & 197 & 164 & \\
\hline Model 1 & 1 & $1.02(0.82,1.27)$ & $0.72(0.58,0.91)$ & $0.51(0.40,0.65)$ & $<0.0001$ \\
\hline Model 2 & 1 & $1.02(0.81,1.29)$ & $0.80(0.63,1.03)$ & $0.63(0.49,0.82)$ & $<0.0001$ \\
\hline Model 3 & 1 & $1.00(0.79,1.28)$ & $0.79(0.61,1.01)$ & $0.62(0.47,0.81)$ & 0.0001 \\
\hline Chronic inflammation $^{\mathrm{a}}$ & 109 & 102 & 76 & 81 & \\
\hline Model 1 & 1 & $0.83(0.62,1.12)$ & $0.57(0.42,0.79)$ & $0.59(0.43,0.81)$ & 0.0003 \\
\hline Model 2 & 1 & $0.82(0.61,1.11)$ & $0.61(0.44,0.84)$ & $0.67(0.48,0.93)$ & 0.008 \\
\hline Model 3 & 1 & $0.81(0.60,1.10)$ & $0.61(0.44,0.84)$ & $0.69(0.49,0.97)$ & 0.01 \\
\hline
\end{tabular}

Model 1: adjusted for age, sex and north/south and urban/rural locations

Model 2: further adjusted for family history of chronic diseases, education, physical activity, smoking, alcohol consumption, energy intake, consumption of carbohydrate and protein (\% of energy) and BMI

Model 3: further adjusted for energy-adjusted dietary GI, red meat, vegetables, fruits, dietary fibre and erythrocyte trans-18:2 fatty acids levels

${ }^{a}$ Number of participants in Q1-Q4 were 777, 777, 776 and 777, respectively. Numbers of cases in each quartile are shown

In stratified analyses, no significant interaction was observed between the trans-18:1 tertiles and sex, age group, region, physical activity levels or central obesity status for type 2 diabetes (ESM Fig. 1a-e). However, the association between trans-18:1 level and diabetes was more pronounced in overweight/obese participants (OR [95\% CI] across extreme [first and third] tertiles $0.56[0.38,0.81] ; p_{\text {trend }}=$ $0.002)$ than in their normal-weight counterparts (1.01 $\left.[0.60,1.72] ; p_{\text {trend }}=0.97\right)\left(\mathrm{ESM}\right.$ Fig. $\left.1 \mathrm{f}, p_{\text {interaction }}=0.008\right)$.

\section{Discussion}

Among more than 3,000 middle-aged and older Chinese individuals, we observed that TFAs accounted for only $0.37 \%$ of the total fatty acids in erythrocytes. trans $-18: 1$, the most abundant type of TFA seen in this population, which consumes a traditional Chinese diet, was significantly associated with dairy consumption. Participants with the highest trans-18:1 levels had about a 30\% lower risk for 
Table 3 ORs of type 2 diabetes and dyslipidaemia according to quartiles of trans-18:2 fatty acids

\begin{tabular}{|c|c|c|c|c|c|}
\hline trans-18:2 & Q1 (median 0.106) & Q2 (median 0.138) & Q3 (median 0.167) & Q4 (median 0.225) & $p$ for trend \\
\hline Type 2 diabetes ${ }^{\mathrm{a}}$ & 112 & 105 & 101 & 107 & \\
\hline Model 1 & 1 & $0.83(0.62,1.12)$ & $0.73(0.54,0.98)$ & $0.78(0.58,1.06)$ & 0.12 \\
\hline Model 2 & 1 & $0.78(0.58,1.05)$ & $0.66(0.49,0.90)$ & $0.71(0.52,0.98)$ & 0.05 \\
\hline Model 3 & 1 & $0.81(0.60,1.10)$ & $0.71(0.52,0.96)$ & $0.76(0.55,1.04)$ & 0.12 \\
\hline Hypertriacylglycerolaemia ${ }^{a, b, c}$ & 104 & 135 & 180 & 201 & \\
\hline Model 1 & 1 & $1.25(0.94,1.66)$ & $1.72(1.31,2.27)$ & $1.88(1.43,2.48)$ & $<0.0001$ \\
\hline Model 2 & 1 & $1.15(0.86,1.55)$ & $1.67(1.26,2.22)$ & $1.91(1.43,2.54)$ & $<0.0001$ \\
\hline Model 3 & 1 & $1.27(0.94,1.71)$ & $1.98(1.48,2.65)$ & $2.34(1.73,3.17)$ & $<0.0001$ \\
\hline High total cholesterol ${ }^{\mathrm{a}, \mathrm{b}, \mathrm{c}}$ & 143 & 177 & 216 & 213 & \\
\hline Model 1 & 1 & $1.18(0.91,1.53)$ & $1.38(1.07,1.78)$ & $1.34(1.03,1.73)$ & 0.03 \\
\hline Model 2 & 1 & $1.15(0.88,1.50)$ & $1.34(1.04,1.74)$ & $1.32(1.02,1.72)$ & 0.04 \\
\hline Model 3 & 1 & $1.15(0.89,1.51)$ & $1.36(1.04,1.76)$ & $1.29(0.98,1.70)$ & 0.05 \\
\hline High LDL-cholesterol ${ }^{a, b, c}$ & 231 & 277 & 323 & 334 & \\
\hline Model 1 & 1 & $1.16(0.92,1.46)$ & $1.33(1.06,1.67)$ & $1.38(1.09,1.74)$ & 0.007 \\
\hline Model 2 & 1 & $1.12(0.88,1.41)$ & $1.27(1.01,1.61)$ & $1.33(1.05,1.68)$ & 0.02 \\
\hline Model 3 & 1 & $1.13(0.89,1.43)$ & $1.29(1.02,1.64)$ & $1.31(1.02,1.68)$ & 0.03 \\
\hline Low HDL-cholesterol ${ }^{\mathrm{a}, \mathrm{b}}$ & 280 & 280 & 290 & 289 & \\
\hline Model 1 & 1 & $1.00(0.80,1.25)$ & $0.99(0.79,1.24)$ & $1.01(0.80,1.27)$ & 0.95 \\
\hline Model 2 & 1 & $0.94(0.74,1.19)$ & $0.93(0.73,1.17)$ & $0.97(0.76,1.24)$ & 0.86 \\
\hline Model 3 & 1 & $0.98(0.78,1.25)$ & $1.00(0.78,1.28)$ & $1.12(0.86,1.44)$ & 0.34 \\
\hline
\end{tabular}

Model 1: adjusted for age, sex and north/south and urban/rural location

Model 2: further adjusted for family history of chronic diseases, education, smoking, alcohol consumption, physical activity and BMI

Model 3: further adjusted for saturated fatty acids and trans-18:1 fatty acids in erythrocytes

${ }^{a}$ Numbers of participants in Q1-Q4 were 694, 694, 693 and 694, respectively. Numbers of cases in each quartile are shown

${ }^{\mathrm{b}}$ Participants $(n=332)$ who took fish oil supplements or hypolipidaemic drugs were excluded

${ }^{\mathrm{c}}$ Defined by the National Centers for Environmental Prediction/Adult Treatment Panel III criteria for borderline high (1.7 mmol/l for triacylglycerol, $5.17 \mathrm{mmol} / \mathrm{l}$ for total cholesterol and $3.4 \mathrm{mmol} / \mathrm{l}$ for LDL-cholesterol)

type 2 diabetes after adjustment for social demographics, lifestyle, BMI, multiple dietary factors and other fatty acids. The associations appeared to be stronger in overweight/ obese individuals. In contrast, increased trans-18:2 levels were associated with a higher risk of dyslipidaemia, independent of conventional risk factors, and erythrocyte saturated and trans-18:1 fatty acids.

To our knowledge, this is the first report on TFA biomarkers in relation to type 2 diabetes and cardiometabolic disorders in a large Asian population. Our participants were sampled from the most economically developed regions of China, although the erythrocyte contents of TFAs were 70$80 \%$ lower than those seen in Western populations, such as the NHS cohort (total TFAs 1.8\%, trans-18:1 1.3\%) using a similar methodology for fatty acid measurement [27]. Even among urban women, who had the highest TFAs of all the subgroups, the mean levels of total TFAs and trans-18:1 were $0.42 \pm 0.12 \%$ and $0.23 \pm 0.06 \%$, respectively, suggesting a low overall intake of TFAs in this Chinese population.

Of note, not only the amount of TFAs, but also the food sources of the TFAs might be different between Chinese and
Western populations. Unlike the CHS, in which ten TFAs in plasma phospholipids were detected from 3,330 US adults, only three isomers of erythrocyte TFAs were detected in the current study. Moreover, trans-18:1 levels in our study were only significantly associated with dairy products among all foods, whereas all trans-18:1 and trans-18:2 isomers in the CHS were associated with PHVO-abundant foods, such as fried foods, margarine and bakery foods [28]. Since TFAs cannot be generated from de novo human synthesis, transisomers in tissues or in the circulation may serve as objective dietary biomarkers [29]. The discrepancies between our investigation and studies conducted in Western populations might be due to a lower consumption of PHVO-rich foods in this 50-70-year-old Chinese population, who mostly cook and eat a traditional Chinese diet at home. In such a diet, dairy products may become the primary source of trans$18: 1$, since dairy fat was reported to contain a substantially higher portion of trans-18:1 than trans-18:2 fatty acids [30].

The moderate association between trans-18:1 and dairy consumption may occur for several reasons. First, the dairy intake in our population (median $40 \mathrm{~g} /$ day) was much lower 
than that in the USA (median $250 \mathrm{~g} /$ day in the US National Health and Nutrition Examination Survey) or in European countries (median $270 \mathrm{~g} /$ day in the Netherlands Cohort Study) [31, 32]. Second, consumption of high-fat dairy products, such as cheese and butter, was almost negligible in our study population. A limited variability of dairy intake levels might lead to a relatively weaker association in the current analysis than has been seen in previous studies [24, 33, 34]. Third, FFQ assessments of less frequently consumed foods are prone to measurement errors. Similarly, measurement errors of less abundant erythrocyte fatty acids tend to be higher. Thus, both sources of measurement error tend to weaken the association [35]. Nonetheless, because the errors in FFQ assessment and erythrocyte fatty acid measurement are independent of each other, the significant correlation supports the use of trans-18:1 as a biomarker of dairy consumption in this population.

In our study, erythrocyte trans-18:1 levels were inversely associated with risk of diabetes and several cardiovascular risk factors. Interestingly, trans-18:1 levels were also related to certain healthy lifestyle and dietary factors, but the associations were basically unchanged after carefully controlling for these confounders and other erythrocyte TFAs, saturated and polyunsaturated fatty acids. Similarly, Mozaffarian et al [13] have reported that trans-16:1n-7 as a unique marker of ruminant fat only represented $0.2 \%$ of total fatty acids in plasma phospholipids and was associated with lower insulin resistance, less dyslipidaemia and a nearly $60 \%$ lower risk of incident diabetes. Because of the overall very low amount of TFAs, trans-16:1 was undetectable in our population. However, previous studies have shown that markers of dairy fat vary in populations with a different dietary background [29]. Accumulating evidence has shown the beneficial effects of dairy products on metabolic syndrome and diabetes [36, 37]. In a recent meta-analysis, the relative risk of diabetes was 0.85 $(0.75,0.96)$ comparing the highest with the lowest dairy consumption [36]. Moreover, milk intake was found to be associated with a lower incidence of type 2 diabetes in the Shanghai Women's Health Study, which involved participants who were comparable with ours in terms of dietary pattern and lifestyle practices [38]. The inverse relationship between dairy-related TFA markers and metabolic disorders observed in our study was consistent with these reports.

Meanwhile, the possible beneficial effects of a particular trans-18:1 isomer - trans-18:1n-7 (vaccenic acid [VA]) have previously been documented. VA supplementation in rats reduced triacylglycerol levels by $40 \%$ compared with a control diet [39]. An antiatherogenic property was also suggested from the administration of VA to LDL-receptordeficient mice [40]. Because the erythrocyte trans-18:1 levels in our study were too low to allow a further distinction between individual trans-18:1 fatty acids in terms of the double-bond location, it is unclear whether the inverse association could be largely due to VA, which is also a TFA found in dairy products [10]. On the other hand, dairy products are a rich source of micronutrients such vitamin $\mathrm{D}$, calcium and magnesium, which have shown cardiometabolic benefits in some studies [37]. In our population, plasma 25-hydroxyvitamin $\mathrm{D}$ level was positively associated with erythrocyte trans-18:1 level $(\beta=0.44, p<0.001)$. However, adjustment for 25-hydroxyvitamin $\mathrm{D}$ did not materially change the inverse associations between trans-18:1 levels and diabetes. Certainly, more studies are needed to elucidate the pathophysiological mechanism(s) of specific isomers and also effects of micronutrients from dairy products on cardiometabolic outcomes.

Consistent with the findings that increased adipose tissue trans-18:1 was associated with decreased BMI and waist circumference in 1,785 Costa Rican individuals [41], we observed that erythrocyte trans-18:1 was associated with lower odds of central obesity. Motard-Bélanger et al [6] reported that a moderate-rTFA diet ( $1.5 \%$ of energy) was able to reduce the waistline and improve blood lipids compared with a control diet. However, it is still unclear whether a trans-configuration could per se modify energy metabolism [42]. Moreover, we observed that the inverse association between trans-18:1 level and type 2 diabetes was more evident among overweight/obese individuals in a stratified analysis. However, mechanistic links between trans-18:1 and obesity and metabolic diseases need to be illuminated in future studies.

Although trans-18:2 isomers in our study only represented less than $0.2 \%$ of the total fatty acids in erythrocytes, the positive associations of these TFAs with blood triacylglycerol, total cholesterol and LDL-cholesterol were robust and independent of established risk factors for dyslipidaemia. Previous studies reported that higher trans-18:2 but lower trans-18:1 levels were associated with greater adiposity [41] and risk of heart disease [12]. However, the influence of unconjugatedtrans-18:2 (trans-18:2n-6 9c12t and 9t12c) on blood lipids and other metabolic disorders needs to be elucidated. In the current study, one of the possible explanations for the opposite associations of the two types of TFA might be that erythrocyte trans-18:1 derived from ruminant fats whereas the trans-18:2 isomers came from PHVOs or transformed linoleic acid (18:2n-6) during frying [12, 43].

Our study has several strengths. This is the first largescale population-based study that has investigated the amounts and food sources of measured TFAs and their associations with cardiometabolic diseases in Chinese men and women. The participants were recruited from both urban and rural areas in northern and southern China. Second, rich data on social-demographic, lifestyle and dietary factors as well as other fatty acids were collected, and major established and potential confounders were extensively adjusted to minimise confounding. Finally, TFA exposure was determined by a widely accepted objective measurement of 
erythrocytes, which have been shown as excellent biomarkers of long-term trans-fat intake [24].

A major limitation of this study is its cross-sectional design, which limits a causal inference. However, because of low levels of health awareness and nutritional education, participants were unlikely to substantially change their diet if they had an elevated BMI or were even diagnosed with hypertension or dyslipidaemia. Nevertheless, in the sensitivity analyses, the overall results were not changed by excluding participants with any previously diagnosed chronic diseases such as hypertension, hypercholesterolaemia or diabetes. Another weakness is that newly developed diabetes was diagnosed by a single measure of fasting plasma glucose level. Misclassification was possible but may have attenuated the association if it were random. In addition, the robust associations with other metabolic biomarkers provide assurance that the observed association with diabetes was less likely to have occurred by chance. Moreover, measurement errors for erythrocyte fatty acids and food intake are inevitable, which may reduce statistical power. In particular, measurement errors for TFAs and dairy consumption may be substantial because of the overall very low levels of these. There might also be other food sources besides dairy products that provide trans-18:1, but these might not have been included in our FFQ, or might have been poorly assessed. Finally, regardless of a wide range of potential confounders that were controlled for, residual or unmeasured confounder(s) may still, to some extent, explain the associations.

In conclusion, erythrocyte trans-18:1 was associated with dairy intake and showed inverse associations with risk of type 2 diabetes and several cardiovascular risk factors in 5070 -year-old Chinese men and women, whereas higher erythrocyte trans-18:2 levels were associated with a higher risk of dyslipidaemia. Our findings may offer novel insights into differential associations between specific TFAs and metabolic disorders in a population with a low consumption of both dairy products and PHVOs. Prospective studies are needed to confirm our findings and clarify the potential underlying mechanisms.

Acknowledgements We are grateful to Z. Yu, L. Chen, Y. Wu, L. Sun, Q. Qi, L. Lu, H. Wu, C. Liu, G. Zhang, S. Ma, H. Zheng and Q. Jin of the Institute for Nutritional Sciences, Chinese Academy of Sciences and Beijing and Shanghai CDC for their kind help at various stages of this study.

Funding This study was funded by grants from the National Basic Research 973 Program (2011CB504002 and 2012CB524900), the National Natural Science Foundation of China (30930081 and 81021002), and the Chinese Academy of Sciences (KSCX2-EW-R-10 and SIBS2008006). Q. Sun is supported by a career development award K99HL098459 from the National Heart, Lung, and Blood Institute.

Duality of interest The authors declare that there is no duality of interest associated with this manuscript.
Contribution statement DXY made substantial contributions to the acquisition and analysis of data and to drafting the article. XWY, AP, HXL, GZ and YHZ made substantial contributions to the acquisition of data and to revising the article critically for important intellectual content. QS and FBH contributed substantially to conception and design, data interpretation and revising the article critically for important intellectual content. XL provided a substantial contribution to the conception and design, to acquisition and interpretation of data and to drafting/revising the article. All authors approved the final version to be published.

\section{References}

1. Hu FB, Liu Y, Willett WC (2011) Preventing chronic diseases by promoting healthy diet and lifestyle: public policy implications for China. Obes Rev 12:552-559

2. Wang Y, Mi J, Shan XY, Wang QJ, Ge KY (2007) Is China facing an obesity epidemic and the consequences? The trends in obesity and chronic disease in China. Int J Obes (Lond) 31:177-188

3. Micha R, Mozaffarian D (2009) Trans fatty acids: effects on metabolic syndrome, heart disease and diabetes. Nat Rev Endocrinol 5:335-344

4. Mozaffarian D, Katan MB, Ascherio A, Stampfer MJ, Willett WC (2006) Trans fatty acids and cardiovascular disease. N Engl J Med 354:1601-1613

5. Mozaffarian D, Aro A, Willett WC (2009) Health effects of transfatty acids: experimental and observational evidence. Eur J Clin Nutr 63(Suppl 2):S5-S21

6. Motard-Bélanger A, Charest A, Grenier G et al (2008) Study of the effect of trans fatty acids from ruminants on blood lipids and other risk factors for cardiovascular disease. Am J Clin Nutr 87:593-599

7. Chardigny JM, Destaillats F, Malpuech-Brugere C et al (2008) Do trans fatty acids from industrially produced sources and from natural sources have the same effect on cardiovascular disease risk factors in healthy subjects? Results of the trans Fatty Acids Collaboration (TRANSFACT) study. Am J Clin Nutr 87:558-566

8. van Dam RM, Willett WC, Rimm EB, Stampfer MJ, Hu FB (2002) Dietary fat and meat intake in relation to risk of type 2 diabetes in men. Diabetes Care 25:417-424

9. Salmeron J, Hu FB, Manson JE et al (2001) Dietary fat intake and risk of type 2 diabetes in women. Am J Clin Nutr 73:1019-1026

10. Morio B, Tardy AL, Chardigny JM, Malpuech-Brugere C (2011) Ruminant and industrial sources of trans-fat and cardiovascular and diabetic diseases. Nutr Res Rev 24:111-117

11. Tholstrup T, Raff M, Basu S, Nonboe P, Sejrsen K, Straarup EM (2006) Effects of butter high in ruminant trans and monounsaturated fatty acids on lipoproteins, incorporation of fatty acids into lipid classes, plasma C-reactive protein, oxidative stress, hemostatic variables, and insulin in healthy young men. Am J Clin Nutr $83: 237-243$

12. Lemaitre RN, King IB, Mozaffarian D et al (2006) Plasma phospholipid trans fatty acids, fatal ischemic heart disease, and sudden cardiac death in older adults: the cardiovascular health study. Circulation 114:209-215

13. Mozaffarian D, Cao H, King IB et al (2010) Trans-palmitoleic acid, metabolic risk factors, and new-onset diabetes in U.S. adults: a cohort study. Ann Intern Med 153:790-799

14. Ye X, Yu Z, Li H, Franco OH, Liu Y, Lin X (2007) Distributions of C-reactive protein and its association with metabolic syndrome in middle-aged and older Chinese people. J Am Coll Cardiol 49:1798-1805

15. Yu Z, Ye X, Wang J et al (2009) Associations of physical activity with inflammatory factors, adipocytokines, and metabolic 
syndrome in middle-aged and older Chinese people. Circulation 119:2969-2977

16. Zhai F, Yang X (eds) (2006) The nutrition and health status of the Chinese people 2002: diet and nutrients intake. People's Medical Publishing House, Beijing

17. Zhao W, Hasegawa K, Chen J (2002) The use of food-frequency questionnaires for various purposes in China. Public Health Nutr 5 (6A):829-833

18. Shi Z, Hu X, Yuan B et al (2006) Association between dietary patterns and anemia in adults from Jiangsu Province in Eastern China. Br J Nutr 96:906-912

19. Yang Y (ed) (2005) Chinese food composition table 2004. Peking University Medical Press, Beijing

20. Atkinson FS, Foster-Powell K, Brand-Miller JC (2008) International tables of glycemic index and glycemic load values: 2008 . Diabetes Care 31:2281-2283

21. American Diabetes Association (2007) Diagnosis and classification of diabetes mellitus. Diabetes Care 30(Suppl 1):S42-S47

22. Grundy SM, Cleeman JI, Daniels SR et al (2005) Diagnosis and management of the metabolic syndrome: an American Heart Association/National Heart, Lung, and Blood Institute Scientific Statement. Circulation 112:2735-2752

23. Pearson TA, Mensah GA, Alexander RW et al (2003) Markers of inflammation and cardiovascular diseases: application to clinical and public health practice. Circulation 107:499-511

24. Sun Q, Ma J, Campos H, Hankinson SE, Hu FB (2007) Comparison between plasma and erythrocyte fatty acid content as biomarkers of fatty acid intake in US women. Am J Clin Nutr 86:74-81

25. Baylin A, Kim MK, Donovan-Palmer A et al (2005) Fasting whole blood as a biomarker of essential fatty acid intake in epidemiologic studies: comparison with adipose tissue and plasma. Am J Epidemiol 162:373-381

26. Willett WC, Howe GR, Kushi LH (1997) Adjustment for total energy intake in epidemiologic studies. Am J Clin Nutr 65:1220S1228S, discussion 1229S-1231S

27. Sun Q, Ma J, Campos H et al (2007) A prospective study of trans fatty acids in erythrocytes and risk of coronary heart disease. Circulation 115:1858-1865

28. Micha R, King IB, Lemaitre RN et al (2010) Food sources of individual plasma phospholipid trans fatty acid isomers: the Cardiovascular Health Study. Am J Clin Nutr 91:883-893

29. Sun Q, Ma J, Campos H, Hu FB (2007) Plasma and erythrocyte biomarkers of dairy fat intake and risk of ischemic heart diseased. Am J Clin Nutr 86:929-937
30. No authors listed (1995) Trans fatty acids and coronary heart disease risk. Report of the expert panel on trans fatty acids and coronary heart disease. Am J Clin Nutr 62: 655S-708S; discussion 518-626

31. Beydoun MA, Gary TL, Caballero BH, Lawrence RS, Cheskin LJ, Wang Y (2008) Ethnic differences in dairy and related nutrient consumption among US adults and their association with obesity, central obesity, and the metabolic syndrome. Am J Clin Nutr 87:1914-1925

32. Goldbohm RA, Chorus AM, Galindo Garre F, Schouten LJ, van den Brandt PA (2011) Dairy consumption and 10-y total and cardiovascular mortality: a prospective cohort study in the Netherlands. Am J Clin Nutr 93:615-627

33. Smedman AE, Gustafsson IB, Berglund LG, Vessby BO (1999) Pentadecanoic acid in serum as a marker for intake of milk fat: relations between intake of milk fat and metabolic risk factors. Am J Clin Nutr 69:22-29

34. Wolk A, Furuheim M, Vessby B (2001) Fatty acid composition of adipose tissue and serum lipids are valid biological markers of dairy fat intake in men. J Nutr 131:828-833

35. Willett W (1998) Nutritional epidemiology. Oxford University Press, New York

36. Elwood PC, Pickering JE, Givens DI, Gallacher JE (2010) The consumption of milk and dairy foods and the incidence of vascular disease and diabetes: an overview of the evidence. Lipids 45:925-939

37. Crichton GE, Bryan J, Buckley J, Murphy KJ (2011) Dairy consumption and metabolic syndrome: a systematic review of findings and methodological issues. Obes Rev 12:e190-e201

38. Villegas R, Gao YT, Dai Q et al (2009) Dietary calcium and magnesium intakes and the risk of type 2 diabetes: the Shanghai Women's Health Study. Am J Clin Nutr 89:1059-1067

39. Wang Y, Lu J, Ruth MR et al (2008) Trans-11 vaccenic acid dietary supplementation induces hypolipidemic effects in JCR:LA-cp rats. J Nutr 138:2117-2122

40. Bassett CM, Edel AL, Patenaude AF et al (2010) Dietary vaccenic acid has antiatherogenic effects in LDLr-/- mice. J Nutr 140:18-24

41. Smit LA, Willett WC, Campos H (2010) Trans-fatty acid isomers in adipose tissue have divergent associations with adiposity in humans. Lipids 45:693-700

42. Thompson AK, Minihane AM, Williams CM (2011) Trans fatty acids and weight gain. Int J Obes (Lond) 35:315-324

43. Sébédio JL, Chardigny JM, Malpuech-Brugère C, Erickson MD (2006) Deep frying chemistry. Physiological effects of trans and cyclic fatty acids: Nutrition and Practical Applications. AOCS Press, Urbana, pp 205-228 